\title{
Addendum: Heliconical smectic phases formed by achiral molecules
}

\author{
Jordan P. Abberley ${ }^{1}$, Ross Killah', Rebecca Walker ${ }^{1}$, John M.D. Storey ${ }^{1}$, Corrie T. Imrie ${ }^{1}$,
} Mirosław Salamończyk (1D ${ }^{2,3}$, Chenhui Zhu², Ewa Gorecka ${ }^{4}$ \& Damian Pociecha ${ }^{4}$

Addendum to: Nature Communications https:/doi.org/10.1038/s41467-017-02626-6; published online 15 Jan 2018

We would like to make our readers aware of the related publications by S.P. Sreenilayam et al. (Nat. Commun. 7, 11369 (2016) and Phys. Rev. Mat. 1, 035604 (2017)), which report the spontaneous helix formation in a polar smectic liquid crystal phase made of achiral bent-core mesogens.

Published online: 17 July 2018

Open Access This article is licensed under a Creative Commons Attribution 4.0 International License, which permits use, sharing, adaptation, distribution and reproduction in any medium or format, as long as you give appropriate credit to the original author(s) and the source, provide a link to the Creative Commons license,

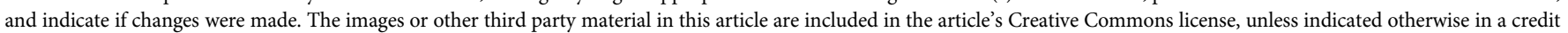

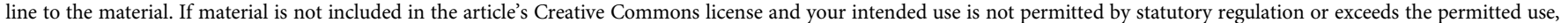
you will need to obtain permission directly from the copyright holder. To view a copy of this license, visit http://creativecommons.org/licenses/by/4.0/.
\end{abstract}

(C) The Author(s) 2018

\footnotetext{
${ }^{1}$ Department of Chemistry, King's College, University of Aberdeen, Aberdeen AB24 3UE, UK. ${ }^{2}$ Advanced Light Source, Lawrence Berkeley National Laboratory, 1 Cyclotron Road, Berkeley, CA 94720, USA. ${ }^{3}$ Department of Physics and Liquid Crystal Institute, Kent State University, 1425 Lefton Esplanade, Kent, OH 44242, USA. ${ }^{4}$ Faculty of Chemistry, University of Warsaw, Zwirki i Wigury 101, Warsaw 02-089, Poland. Correspondence and requests for materials should be addressed to D.P. (email: pociu@chem.uw.edu.pl)
} 